\title{
ON SOME PROBLEMS RELATED TO PALINDROME CLOSURE*
}

\author{
Michelangelo Bucci ${ }^{1}$, Aldo De LucA ${ }^{1}$, \\ Alessandro De LuCA $^{1}$ And LucA Q. Zamboni ${ }^{2}$
}

\begin{abstract}
In this paper, we solve some open problems related to (pseudo)palindrome closure operators and to the infinite words generated by their iteration, that is, standard episturmian and pseudostandard words. We show that if $\vartheta$ is an involutory antimorphism of $A^{*}$, then the right and left $\vartheta$-palindromic closures of any factor of a $\vartheta$-standard word are also factors of some $\vartheta$-standard word. We also introduce the class of pseudostandard words with "seed", obtained by iterated pseudopalindrome closure starting from a nonempty word. We show that pseudostandard words with seed are morphic images of standard episturmian words. Moreover, we prove that for any given pseudostandard word $s$ with seed, all sufficiently long left special factors of $s$ are prefixes of it.
\end{abstract}

Mathematics Subject Classification. 68R15.

\section{INTRODUCTION}

Sturmian words are a classical subject of combinatorics on words (see for instance [3]); by definition, they are infinite words having $n+1$ factors (i.e., blocks of consecutive symbols) of each length $n$. Sturmian words enjoy many interesting characterizations and have a wide range of applications, from discrete geometry to crystallography.

Palindrome closure operators, introduced in [5], have had an important role in the study of Sturmian words. If $w$ is a word, its right (resp. left) palindrome closure

Keywords and phrases. Palindromes, palindrome closures, Sturmian and episturmian words, involutory antimorphisms, pseudopalindromes, pseudostandard words.

* The work for this paper has been supported by the Italian Ministry of Education under Project COFIN 2005 - Automi e Linguaggi Formali: aspetti matematici e applicativi.

1 Dipartimento di Matematica e Applicazioni "R. Caccioppoli", Università degli Studi di Napoli Federico II. Via Cintia, Monte S. Angelo, I-80126 Napoli, Italy; micbucci@unina.it; aldo.deluca@unina.it; alessandro.deluca@unina.it

2 Department of Mathematics, PO Box 311430, University of North Texas. Denton TX, USA; luca@unt.edu 
$w^{(+)}\left(\right.$resp. $\left.w^{(-)}\right)$is the shortest palindrome having $w$ as a prefix (resp. suffix). Standard Sturmian words can be constructed by iterated palindrome closure, that is, by the following procedure. Start from the empty word, and successively add a letter from $\{a, b\}$ and apply the right palindrome closure operator. In this way one generates a sequence of palindromes, each one being a prefix of the next one, so that a limit is naturally defined. If both $a$ and $b$ are used infinitely many times during such process, the infinite word obtained as a limit is aperiodic, and is exactly a standard Sturmian word. For any Sturmian word, there exists a (unique) standard Sturmian word having the same factors.

In recent years, many extensions of Sturmian words have appeared. In particular, in [7] episturmian words were introduced (see also [10]). They can be defined as words having the same set of factors of a standard episturmian word, which is just a word obtained by iterated palindrome closure over an arbitrary alphabet (and without the aperiodicity condition).

A further generalization was introduced in [6], by substituting palindrome closure with pseudopalindrome closure. A pseudopalindrome is a fixed point of some involutory antimorphism $\vartheta$ of a free monoid $A^{*}$. Thus ordinary palindromes are a special case of pseudopalindromes where the antimorphism is simply the reversal operator. We speak of $\vartheta$-palindromes when a particular antimorphism $\vartheta$ is chosen. It is then natural to consider $\vartheta$-palindrome closure operators, and to look at words obtained by iterated $\vartheta$-palindrome closure, called $\vartheta$-standard (or generally pseudostandard) words.

In this paper, we discuss some properties related to (pseudo)palindrome closure, episturmian and $\vartheta$-standard words. In [6] it was proven that both palindromic closures $w^{(+)}$and $w^{(-)}$of a factor $w$ of a Sturmian word are themselves factors of Sturmian words. In Section 2, this property is proved for episturmian words.

In Section 3, the closure property is extended to factors of $\vartheta$-standard words too. We also show that a $\vartheta$-standard word having both closures as factors always exists. Moreover, we prove that every left special factor of a $\vartheta$-standard word $t$, whose length is at least 3 , is a prefix of $t$. Recall that a factor $u$ of a (finite or infinite) word $w$ over an alphabet $A$ is left (resp. right) special if there exist at least two distinct letters $a, b \in A$ such that both $a u$ and $b u$ (resp. $u a$ and $u b$ ) are factors of $w$.

In the last section we introduce the class of $\vartheta$-standard words with seed. They are infinite words obtained by iterated $\vartheta$-palindrome closure, starting from an arbitrary word $u_{0}$ (called seed) instead of the empty word. We show that every $\vartheta$-standard word with seed is a morphic image of a standard episturmian word. More precisely, if $\Delta=x x_{1} x_{2} \ldots x_{n} \ldots$ is the infinite sequence of letters which directs the construction of a $\vartheta$-standard word $t$ with a seed, then $t=\phi_{x}(s)$, where $\phi_{x}$ is a morphism depending on $\vartheta$ and $u_{0}$, and $s$ is the standard episturmian word directed by $\Delta^{\prime}=x_{1} x_{2} \ldots x_{n} \ldots$

Finally, we show that every sufficiently long left special factor of a $\vartheta$-standard word with seed is a prefix of it, and give an upper bound for the minimal length from which this occurs, in terms of the length of the right $\vartheta$-palindrome closure of $u_{0} x$. This proves a conjecture posed in [6]. 


\section{Preliminaries}

Let $A$ be a finite alphabet and $A^{*}$ the free monoid generated by $A$. The elements of $A$ are usually called letters and those of $A^{*}$ words. The identity element of $A^{*}$ is called empty word and denoted by $\varepsilon$. We set $A^{+}=A^{*} \backslash\{\varepsilon\}$. A word $w \in A^{+}$ can be written uniquely as a sequence of letters $w=a_{1} a_{2} \ldots a_{n}$, with $a_{i} \in A$, $i=1, \ldots, n$. The integer $n$ is called the length of $w$ and is denoted by $|w|$. The length of $\varepsilon$ is conventionally 0 .

Let $w \in A^{*}$. A word $v$ is a factor of $w$ if there exist words $r$ and $s$ such that $w=r v s$. If $w=v s$ for some $s$ (resp. $w=r v$ for some word $r$ ), then $v$ is called a prefix (resp. a suffix) of $w$. A word which is both a prefix and a suffix of $w$ is called a border of $w$. We shall denote respectively by $\operatorname{Fact}(w), \operatorname{Pref}(w)$, and $\operatorname{Suff}(w)$ the sets of all factors, prefixes, and suffixes of the word $w$.

For $X \subseteq A^{*}$ and $u \in A^{*}, u^{-1} X$ and $X u^{-1}$ denote respectively the sets

$$
\left\{w \in A^{*} \mid u w \cap X \neq \emptyset\right\} \text { and }\left\{w \in A^{*} \mid w u \cap X \neq \emptyset\right\} .
$$

When $X$ is a singleton $\{x\}$ and $u^{-1} X \neq \emptyset$ (resp. $X u^{-1} \neq \emptyset$ ), the unique word $w \in u^{-1}\{x\}$ (resp. $w \in\{x\} u^{-1}$ ) is denoted by $u^{-1} x$ (resp. $x u^{-1}$ ).

If $w=a_{1} \ldots a_{n} \in A^{*}, a_{i} \in A, i=1, \ldots, n$, the reversal, or mirror image, of $w$ is the word

$$
\tilde{w}=a_{n} \ldots a_{1} .
$$

One sets $\tilde{\varepsilon}=\varepsilon$. A word is called palindrome if it is equal to its reversal. Any border of a palindrome is trivially a palindrome. We shall denote by $\operatorname{PA} L(A)$, or simply $P A L$, the set of all palindromes on the alphabet $A$.

An infinite word (from left-to-right) $x$ over the alphabet $A$ is any map $x$ : $\mathbb{N}_{+} \longrightarrow A$ where $\mathbb{N}_{+}$is the set of positive integers. We can represent $x$ as

$$
x=x_{1} x_{2} \ldots x_{n} \ldots,
$$

where for any $i>0, x_{i}=x(i) \in A$. A (finite) factor of $x$ is either the empty word or any sequence $u=x_{i} \ldots x_{j}$ with $i \leq j$, i.e., any block of consecutive letters of $x$. If $i=1$, then $u$ is a prefix of $x$. We shall denote by $\operatorname{Fact}(x)$ and $\operatorname{Pref}(x)$ the sets of finite factors and prefixes of $x$ respectively. The set of all infinite words over $A$ is denoted by $A^{\omega}$. Moreover, we set $A^{\infty}=A^{*} \cup A^{\omega}$.

The product between a finite word $w$ and an infinite one $x$ is naturally defined as the infinite word $w x$. An occurrence of the word $v$ in $w \in A^{\infty}$ is any pair $(r, s)$, with $r \in A^{*}$ and $s \in A^{\infty}$, such that $w=r v s$. If $w \in A^{*}$ and $a \in A,|w|_{a}$ denotes the number of distinct occurrences of $a$ in $w$.

If $x \in A$ and $v x$ (resp. $x v$ ) is a factor of $w \in A^{\infty}$, then $v x$ (resp. $x v$ ) is called a right (resp. left) extension of $v$ in $w$. We recall that a factor $v$ of a (finite or infinite) word $w$ is called right special if it has at least two distinct right extensions in $w$, i.e., there exist at least two distinct letters $a, b \in A$ such that both $v a$ and $v b$ are factors of $w$. Left special factors are defined analogously. A factor of $w$ is called bispecial if it is both right and left special. 
We call a factor $w$ of a word $s \in A^{\infty}$ a first return to $u$ if $w$ contains exactly two distinct occurrences of $u$, one as a prefix and the other as a suffix, i.e.,

$$
w=u \lambda=\mu u \quad \text { with } \lambda, \mu \in A^{+} \text {and } w \notin A^{+} u A^{+} .
$$

We observe that in such a case, $w u^{-1}=\mu$ is usually called a return word over $u$ in $s$ (see [8]).

A word $s \in A^{\omega}$ is said to be closed under reversal if for any $u \in \operatorname{Fact}(s)$ one has $\tilde{u} \in \operatorname{Fact}(s)$. In this case, a factor $u$ of $s$ is right special if and only if $\tilde{u}$ is a left special factor of $s$.

A word $w \in A^{\omega}$ is called episturmian if it is closed under reversal and it has at most one right (or equivalently, left) special factor of each length. We recall (see [7]) that every episturmian word is uniformly recurrent, i.e., every factor of an episturmian word occurs infinitely often, with bounded gaps.

An episturmian word $w$ is called standard if every left special factor of $w$ is a prefix of it. We denote by $E p(A)$, or simply $E p$, the set of all episturmian words over $A$, and by $S E p$ the set of standard ones.

Proposition 1.1 (cf. [7]). For every episturmian word $w$, there exists a standard episturmian word s such that $\operatorname{Fact}(s)=\operatorname{Fact}(w)$.

Thus $\operatorname{Fact}(E p)=\operatorname{Fact}(S E p)$. The elements of Fact $(E p)$ are called finite episturmian words.

Given a word $w \in A^{*}$, we denote by $w^{(+)}$its right palindrome closure, i.e., the shortest palindrome having $w$ as a prefix. Similarly, $w^{(-)}$is the left palindrome closure of $w$. For instance, if $w=a b a c b c a$, then $w^{(+)}=a b a c b c a b a$ and $w^{(-)}=$ acbcabacbca.

For any $w \in A^{*}$, one has $w^{(-)}=\tilde{w}^{(+)}$. Moreover, if $Q$ is the longest palindromic suffix of $w$ and $w=s Q$, then $w^{(+)}=s Q \tilde{s}$.

Let $\psi: A^{*} \rightarrow A^{*}$ be defined by $\psi(\varepsilon)=\varepsilon$ and $\psi(v a)=(\psi(v) a)^{(+)}$for any $a \in A$ and $v \in A^{*}$. For any $u, v \in A^{*}$, one has $\psi(u v) \in \psi(u) A^{*} \cap A^{*} \psi(u)$. The map $\psi$ can then be naturally extended to $A^{\omega}$ by setting, for any infinite word $x$,

$$
\psi(x)=\lim _{n \rightarrow \infty} \psi\left(w_{n}\right)
$$

where $\left\{w_{n}\right\}=\operatorname{Pref}(x) \cap A^{n}$ for all $n \geq 0$.

Proposition 1.2 (cf. [7]). Let $s \in A^{\omega}$. The following conditions are equivalent:

(1) $s$ is a standard episturmian word,

(2) for any prefix $u$ of $s, u^{(+)}$is also a prefix of $s$,

(3) there exists $x \in A^{\omega}$ such that $s=\psi(x)$.

Given a standard episturmian word $s$, the (unique) infinite word $x$ such that $s=\psi(x)$ is called directive word of $s$ and is denoted by $\Delta(s)$, or simply by $\Delta$. From the preceding proposition, one can easily derive $(c f .[7])$ that the set of palindromic prefixes of a standard episturmian word $s$ coincides with

$$
\{\psi(u) \mid u \in \operatorname{Pref}(\Delta(s))\} .
$$


A standard episturmian word $s$ over the alphabet $A$ is called a (standard) ArnouxRauzy word if every symbol of $A$ occurs infinitely often in the associated directive word $\Delta(s)$. We will denote by $A R(A)$, or simply $A R$, the set of Arnoux-Rauzy words over $A$. In the case of a binary alphabet, an $A R$-word is usually called standard Sturmian word.

Example 1.3. Let $A=\{a, b\}$ and $x=(a b)^{\omega}$. One has that

$$
f=\psi(x)=a b a a b a b a a b a a b a b a \ldots
$$

is the famous Fibonacci word, a standard Sturmian word. On an alphabet with three letters $A=\{a, b, c\}$, if we take $x=(a b c)^{\omega}$ as a directive word, then

$$
\tau=\psi(x)=\text { abacabaabacababacabaabac } \ldots
$$

is a standard Arnoux-Rauzy word, often called Tribonacci word. The word $s=$ cabaabacababacabaab... such that abas $=\tau$ is an example of an episturmian word which is not standard, as $a$ is a left special factor of $s$ but not a prefix of it.

The periodic word $s=(a b a c)^{\omega}$ is standard episturmian, but not Arnoux-Rauzy. Its directive word is $\Delta(s)=a b c^{\omega}$.

The following proposition can be easily proved using well-known results on episturmian words (see [7]).

Proposition 1.4. Let $s$ be a standard episturmian word. Any bispecial factor of $s$ is a palindromic prefix of $s$. If $s$ is not periodic, the converse holds too.

Proposition 1.5. Fact $(E p)=\operatorname{Fact}(A R)$.

Proof. Let $u \in \operatorname{Fact}(E p)=\operatorname{Fact}(S E p)$. Hence there exists $s \in S E p$ such that $u \in \operatorname{Fact}(s)$. Now let be $s=\psi(\Delta)$ where $\Delta=t_{1} t_{2} \ldots t_{n} \ldots$, with $t_{i} \in A$ for $i \geq 1$. Therefore there exists a palindromic prefix $p$ of $s$ such that $u \in \operatorname{Fact}(p)$. Now $p=\psi\left(t_{1} \ldots t_{i}\right)$ for some $i$. We can consider $\Delta^{\prime}=t_{1} \ldots t_{i} t$ with $t \in A^{\omega}$ such that any letter of $A$ occurs infinitely many times in $t$. Hence $s^{\prime}=\psi\left(\Delta^{\prime}\right) \in A R$ and contains $p$ as a factor, so that $u \in \operatorname{Fact}\left(s^{\prime}\right)$. Therefore, $\operatorname{Fact}(E p) \subseteq \operatorname{Fact}(A R)$. Since the inverse inclusion is trivial, the result follows.

The following proposition collects two properties of standard episturmian words (cf. Lems. 1 and 4 in [7]) which will be useful in the sequel.

Proposition 1.6 (cf. [7]). Let $s$ be a standard episturmian word. The following hold:

(1) Any prefix $p$ of $s$ has a palindromic suffix which has a unique occurrence in $p$.

(2) The first letter of s occurs in every factor of s having length 2.

Clearly, if $p$ is a prefix of a standard episturmian word, then the palindromic suffix of $p$ which has a unique occurrence in $p$ is the longest palindromic suffix of $p$. 


\section{A Closure property}

We want to show that if $w \in \operatorname{Fact}(E p)$, then also its right and left palindrome closures belong to Fact $(E p)$; since episturmian words are closed under reversal, and $w^{(-)}=\tilde{w}^{(+)}$, it suffices to prove only the right palindrome closure case. We have the following:

Proposition 2.1. Let $u$ be a non-palindromic finite episturmian word; let $Q$ be the longest palindromic suffix of $u$ and write $u=s a Q$ where $a \in A$ and $s \in A^{*}$ ( $s$ possibly empty). Then ua = saQa is a finite episturmian word.

Before proving the proposition we need some lemmas. The first lemma was proved in [1], Theorem 1.1. We report here a different and simpler proof.

Lemma 2.2. Let $w$ be an episturmian word and $P \in P A L \cap \operatorname{Fact}(w)$. Then every first return to $P$ in $w$ is a palindrome.

Proof. By Proposition 1.1, we may always suppose that $w$ is a standard episturmian word. Let $u \in \operatorname{Fact}(w)$ be a first return to the palindrome $P$, i.e., $u=P \lambda=\rho P, \lambda, \rho \in A^{*}$, and the only two occurrences of $P$ in $u$ are as a prefix and as a suffix of $u$. If $|P|>|\rho|$, then the prefix $P$ of $u$ overlaps with the suffix $P$ in $u$ and this implies, as is easily to verify, that $u$ is a palindrome. Then let us suppose that $u=P v P$ with $v \in A^{*}$.

Now we consider the first occurrence of $u$ or of $\tilde{u}$ in $w$. Without loss of generality, we may suppose that $w=\alpha u w^{\prime}$ and that $\tilde{u}$ does not occur in the prefix of $w$ having length $|\alpha u|-1$. Let $Q$ be the palindromic suffix of $\alpha u$ of maximal length. If $|Q|>|u|$, then we have that $\tilde{u}$ occurs in $\alpha u$ before $u$, which is absurd. Then suppose $|Q| \leq|u|$. If $|u|>|Q|>|P|$, then one contradicts the hypothesis that $u$ is a first return to $P$. If $|Q|=|P|$, then $Q=P$ has more than one occurrence in $\alpha u$, which is absurd in view of Proposition 1.6. The only remaining possibility is $Q=u$, i.e., $u$ is a palindrome.

The following lemma is well-known. We report here a proof for the sake of completeness.

Lemma 2.3. Let $w \in A R$ and $s$ be the unique right special factor of length $n$. If $B_{1}, \ldots, B_{m}, \ldots$ are the bispecial factors of $w$ ordered by increasing length, then $s$ is a suffix of any $B_{m}$ such that $|s| \leq\left|B_{m}\right|$ and, for any $x \in A$, sx $\in \operatorname{Fact}(w)$.

Proof. Since $w$ is not periodic, by Proposition 1.4 the bispecial factors $B_{i}, i>0$, are its palindromic prefixes. Moreover, if $t=t_{1} t_{2} \ldots t_{n} \ldots \in A^{\omega}$ is the directive word of $w$, then $B_{i+1}=\left(B_{i} t_{i}\right)^{(+)}$for any $i>0$. Since $s$ is a right special factor of $w, \tilde{s}$ is left special and thus a prefix of $w$. Therefore, $s$ is a suffix of any palindromic prefix $B_{m}$ of $w$ such that $|s| \leq\left|B_{m}\right|$. As $w \in A R$, any letter $x \in A$ occurs infinitely often in $t$; hence there exists $k \geq m$ such that $x=t_{k}$, so that $B_{k} x$ is a factor of $w$. Since $B_{m}$ is a suffix of $B_{k}$, it follows $s x \in \operatorname{Fact}(w)$.

Lemma 2.4. Let $w$ and $w^{\prime}$ be Arnoux-Rauzy words on the alphabet A. If $w$ and $w^{\prime}$ have the same right special factor of length $n$, then they share the same factors up to length $n+1$. 
Proof. Trivial if $n=0$. By induction, suppose we have proved the assertion for the integer $n-1 \geq 0$. Let $Q$ be the common right special factor of $w$ and $w^{\prime}$ of length $n$. If we write $Q=a Q^{\prime}$, with $a \in A$, then $Q^{\prime}$ is the only right special factor of length $n-1$ of both $w$ and $w^{\prime}$. Hence $w$ and $w^{\prime}$ have the same factors up to length $n$.

By symmetry, it suffices to prove that any factor $v$ of $w$, of length $|v|=n+1$, is also a factor of $w^{\prime}$. Let $v=v^{\prime} b, b \in A$. Suppose first that $v^{\prime}=Q$. By Lemma 2.3, each right extension $Q x$, with $x \in A$, is a factor of both $w$ and $w^{\prime}$; in particular, $v$ is a factor of $w^{\prime}$.

Now assume that $v^{\prime} \neq Q$. Let $v^{\prime}=c v^{\prime \prime}$ with $c \in A$, and suppose that $v^{\prime \prime}=Q^{\prime}$. One has then $c \neq a$. In this case, since $v=c v^{\prime \prime} b$ and $Q b=a v^{\prime \prime} b$ are different factors of $w$, one has that $v^{\prime \prime} b$ is left special in $w$. Since $\left|v^{\prime \prime} b\right|=n$, one derives that $v^{\prime \prime} b=\tilde{Q}$ is a left special factor of $w^{\prime}$ too, so that $v=c v^{\prime \prime} b$ is a factor of $w^{\prime}$ as a consequence of Lemma 2.3.

If $v^{\prime \prime} \neq Q^{\prime}$, then $v^{\prime \prime} b$ is the unique right extension of $v^{\prime \prime}$ in $w$. As $\left|v^{\prime \prime} b\right|=n$, it is also a factor of $w^{\prime}$, and no other letter $x$ is such that $v^{\prime \prime} x \in \operatorname{Fact}\left(w^{\prime}\right)$. Hence $v=c v^{\prime \prime} b$ is the only right extension in $w^{\prime}$ of the factor $c v^{\prime \prime} \neq Q$.

We can now proceed to prove Proposition 2.1.

Proof of Proposition 2.1. We first observe that $u$ contains a single occurrence of $Q$. Indeed, if $u$ contained other occurrences of $Q$, by Lemma 2.2 the suffix of $u$ beginning with the penultimate occurrence would be a palindromic suffix of $u$ strictly longer than $Q$, contradicting the hypothesis of maximality of the length of $Q$.

By Proposition 1.5 there exists an Arnoux-Rauzy word $w$ such that $u \in \operatorname{Fact}(w)$. We can assume that $u a \notin \operatorname{Fact}(w)$ (otherwise $u a$ is in $\operatorname{Fact}(A R)$ as required); so there exist $b \in A$ such that $b \neq a$ and $u b \in \operatorname{Fact}(w)$. Thus $a Q b \in \operatorname{Fact}(w)$; since $Q$ is a palindrome and $w \in A R$, also $b Q a \in \operatorname{Fact}(w)$ and $Q$ is a bispecial factor of $w$. Then it follows that every left special factor of $w$ longer that $Q$ must contain $Q$ as a prefix, and since there is only a single occurrence of $Q$ in $u, Q$ itself is the longest suffix of $u$ which is left special in $w$. Thus every occurrence of $a Q$ in $w$ must be "preceded" by $s$, i.e., if $w=\lambda a Q \mu$, then $w=\lambda^{\prime} s a Q \mu$, with $\lambda=\lambda^{\prime} s$. In particular $a Q a$ is not a factor of $w$, for otherwise $u a$ would be in $\operatorname{Fact}(w)$, contradicting our assumption.

Set $\Delta(w)=t_{1} t_{2} \ldots$ Let $B_{1}=\varepsilon, B_{2}, \ldots$ be the sequence of all bispecial factors of $w$, ordered by increasing length, i.e., $\left|B_{i}\right|<\left|B_{i+1}\right|$ for all $i>0$. By Proposition 1.4, they are the palindromic prefixes of $w$ as $w$ is not periodic. Moreover, for each $i>0$ we have $B_{i+1}=\left(B_{i} t_{i}\right)^{(+)}$, so that $B_{i} t_{i}$ is left special and $t_{i} B_{i}$ is right special.

Since $Q$ is a bispecial factor of $w$, one has $Q=B_{m}$ for some $m>1$. Let $|Q|=$ $n-1$ for $n \geq 2$. We then have that $t_{m} Q$ is right special in $w$ and, from Lemma 2.3, $t_{m} Q x \in \operatorname{Fact}(w)$ for all $x \in A$. It is clear that $t_{m} \neq a$ since $a Q a \notin \operatorname{Fact}(w)$ and $t_{m} Q a \in \operatorname{Fact}(w)$, then we have that $a Q b$ and $t_{m} Q b$ are distinct factors of $w$, thus $Q b$ is left special and $b Q$ is the unique right special factor of $w$ of length $n$. So $t_{m}=b$.

Let $w^{\prime}$ be any Arnoux-Rauzy sequence over $A$, whose directive word $\Delta\left(w^{\prime}\right)=$ $t_{1}^{\prime} t_{2}^{\prime} \ldots$ satisfies $t_{i}^{\prime}=t_{i}$ for $0<i \leq m-1$ and $t_{m}^{\prime}=a$. Since $Q$ is the unique right 
special factor of $w$ and $w^{\prime}$ of length $n-1$, from Lemma 2.4, we obtain that $w$ and $w^{\prime}$ have the same factors of length $k$ for each $k \leq n$. However, they differ on some factors of length $n+1$. Indeed, from the definition of $w^{\prime}$, we have that $a Q$ is its unique right special factor of length $n$, so that by Lemma 2.3, for all $x \in A$ we have that $a Q x \in \operatorname{Fact}\left(w^{\prime}\right)$. Therefore $a Q a \in \operatorname{Fact}\left(w^{\prime}\right) \backslash \operatorname{Fact}(w)$.

Now let us prove that, as in $w$, each occurrence of $a Q$ in $w^{\prime}$ is preceded by $s$. Let $p \in A^{*}$ be such that $|p|=|s|$ and $p a Q \in \operatorname{Fact}\left(w^{\prime}\right)$. Let then $S$ be the largest common suffix of $p a Q$ and $s a Q$ and $Q^{\prime}$ its prefix of length $n-1$. Clearly $Q \neq Q^{\prime}$ since there is only one occurrence of $Q$ in $s a Q$. If we assume that $S \neq p a Q$, then there exist $x, y \in A$ such that $x \neq y, x S \in \operatorname{Suff}(s a Q)$ and $y S \in \operatorname{Suff}(p a Q)$; then $x Q^{\prime}$ and $y Q^{\prime}$ are both factors of $w$ and $w^{\prime}$ since these latter words have the same factors of length $n$. Thus $Q^{\prime}$ is a left special factor of $w$ and $w^{\prime}$, and that is a contradiction, since the only left special factor of length $n-1$ in $w$ and in $w^{\prime}$ is $Q$. Thus $p=s$ and so every occurrence of $a Q$ in $w^{\prime}$ is preceded by $s$.

Since $a Q a$ is a factor of $w^{\prime}$, it follows that $s a Q a=u a$ is a factor of $w^{\prime}$. Hence $u a$ is in $\operatorname{Fact}(A R)$ as required.

From the preceding proposition one derives the following theorem, announced without proof in [6].

Theorem 2.5. If $w$ is a finite episturmian word, then so is each of $w^{(+)}$and $w^{(-)}$.

Proof. Trivial if $w \in P A L$. Let then $w=a_{1} \ldots a_{n} Q$, where $a_{i} \in A$ for $i=$ $1, \ldots, n$ and $Q$ is the longest palindromic suffix of $w$. By Proposition 2.1, $w a_{n}=$ $a_{1} \ldots a_{n} Q a_{n}$ is a finite episturmian word; since its longest palindromic suffix is $a_{n} Q a_{n}$, also $w a_{n} a_{n-1}$ is episturmian. In this way, by applying Proposition 2.1 exactly $n$ times, one eventually obtains that

$$
a_{1} a_{2} \ldots a_{n} Q a_{n} \ldots a_{2} a_{1}=w^{(+)}
$$

is episturmian. Since $w^{(-)}=\tilde{w}^{(+)}$, the assertion follows.

Corollary 2.6. Let $a \in A$ and $u \in A^{*}$. If au is a finite episturmian word, then so is $a u^{(+)}$.

Proof. If $a u$ is not a palindrome, then by Theorem 2.5, $(a u)^{(+)}=a u^{(+)} a$ is an episturmian word and therefore so is $a u^{(+)}$. Let us then suppose that $a u$ is a palindrome.

By Theorem 2.5 one has $u^{(+)} \in \operatorname{Fact}(s)$ for a suitable $s \in A R$. Since $s$ is recurrent there exist letters $x, y \in A$ such that

$$
x u^{(+)} y \in \operatorname{Fact}(s)
$$

If $x \neq y$, then, since $s$ is closed under reversal, one has also $y u^{(+)} x \in \operatorname{Fact}(s)$. Hence $u^{(+)}$is bispecial, so that it follows $a u^{(+)} \in \operatorname{Fact}(s)$. Let us now consider the case $x=y$. If $x=a$, then the assertion is trivially verified. 
Suppose then $x \neq a$. As $a u$ is a palindrome, we can write $u=u^{\prime} a$ with $u^{\prime} \in P A L$. Hence,

$$
x\left(u^{\prime} a\right)^{(+)} x \in \operatorname{Fact}(s) .
$$

Since $\left(u^{\prime} a\right)^{(+)}$begins with $u^{\prime} a$ and ends with $a u^{\prime}$, one has that $x u^{\prime} a$ and $a u^{\prime} x$ are factors of $s$, so that $u^{\prime}$ is bispecial and then a palindromic prefix of $s$ by Proposition 1.4.

Let $\Delta(s)=t_{1} t_{2} \ldots t_{n} \ldots$ be the directive word of $s$. There exists an integer $k$ such that $u^{\prime}=\psi\left(t_{1} t_{2} \ldots t_{k}\right)$. We consider any $A R$ word $s^{\prime}$ whose directive word $\Delta\left(s^{\prime}\right)$ has the prefix $t_{1} t_{2} \ldots t_{k} a$. Thus $u^{\prime} a=u$ is a prefix of $s^{\prime}$. This implies, by Propositions 1.2 and 1.4, that $u^{(+)}$is a bispecial prefix of $s^{\prime}$. From this one derives $a u^{(+)} \in \operatorname{Fact}\left(s^{\prime}\right)$.

\section{Pseudostandard words}

An involutory antimorphism of the free monoid $A^{*}$ is a map $\vartheta: A^{*} \rightarrow A^{*}$ such that $\vartheta(u v)=\vartheta(v) \vartheta(u)$ for any $u, v \in A^{*}$, and $\vartheta \circ \vartheta=\mathrm{id}$. The reversal operator

$$
R: w \in A^{*} \mapsto \tilde{w} \in A^{*}
$$

is the basic example of involutory antimorphism of $A^{*}$. Any involutory antimorphism is the composition $\vartheta=\tau \circ R=R \circ \tau$ where $\tau$ is an involutory permutation of the alphabet $A$. Thus it makes sense to call $\vartheta$-palindromes the fixed points of an involutory antimorphism $\vartheta$. We shall denote by $P A L_{\vartheta}$ the set of $\vartheta$-palindromes over $A$. One can then define the $\vartheta$-palindrome closure operators: $w^{\oplus \vartheta}$ (resp. $w^{\ominus \vartheta}$ ) denotes the shortest $\vartheta$-palindrome having $w$ as a prefix (resp. suffix).

Some properties and results relating $\vartheta$-palindrome closure operators with periodicity and conjugacy are in [6]. Further interesting combinatorial properties of $\vartheta$-palindromes, motivated by problems of molecular biology, have been recently studied in [11].

In the following, we shall fix an involutory antimorphism $\vartheta$ of $A^{*}$, and use the notation $\bar{w}$ for $\vartheta(w)$. We shall also drop the subscript $\vartheta$ from the $\vartheta$-palindrome closure operator $\oplus_{\vartheta}$ when no confusion arises. If $w=s Q=P t$, where $Q$ (resp. $P$ ) is the longest $\vartheta$-palindromic suffix (resp. prefix) of $w$, then

$$
w^{\oplus}=s Q \bar{s} \quad \text { and } \quad w^{\ominus}=\bar{t} P t
$$

(see [6]). Moreover, from the definition it follows

$$
w^{\ominus}=\bar{w}^{\oplus}
$$

for any $w \in A^{*}$.

For example, when $A=\{a, b\}, \vartheta=E \circ R$ where $E$ is the interchange morphism defined by $E(a)=b$ and $E(b)=a$, one has $(a a b a b)^{\oplus}=a a b a b b$ and $(a a b a b)^{\ominus}=$ $a b a b b a a b a b$.

The following lemma will be useful in the sequel. 
Lemma 3.1. For any $u \in P A L_{\vartheta} \backslash\{\varepsilon\}$ and $a \in A,(u a)^{\oplus}$ is a first return to $u$, i.e., if $(u a)^{\oplus}=\lambda u \rho$ with $\lambda, \rho \in A^{*}$, then either $\lambda=\varepsilon$ or $\rho=\varepsilon$.

Proof. By contradiction, let $\lambda, \rho \in A^{+}$be such that

$$
(u a)^{\oplus}=\lambda u \rho .
$$

Clearly $|\lambda|+|u|+|\rho|=\left|(u a)^{\oplus}\right| \leq 2|u|+2$, which implies $|\lambda| \leq|u|+2-|\rho| \leq|u|+1$. Let us show that actually one has $|\lambda| \leq|u|$. Indeed, if $\lambda=u a$ then from (1) one derives $\left|(u a)^{\oplus}\right|=2|u|+2$; this implies that $a \notin P A L_{\vartheta}$ and $(u a)^{\oplus}=u a \bar{a} u=u a u \rho$, so that $u \rho=\bar{a} u$. It follows that for some $k>0, u=\bar{a}^{k} \notin P A L_{\vartheta}$, a contradiction.

Let then $v, w \in A^{*}$ be such that $u=\lambda v$ and $(u a)^{\oplus}=u w=\bar{w} u$, whence $\lambda u \rho=$ $u w=\lambda v w$. Thus $u \rho=v w$, so that $v$ is also a prefix of $u$ and therefore a border of $u$. Since $u$ is a $\vartheta$-palindrome, $v$ is a $\vartheta$-palindrome too, so that $u=\lambda v=v \bar{\lambda}$. Therefore

$$
(u a)^{\oplus}=\lambda u \rho=\lambda v \bar{\lambda} \rho .
$$

Thus $\lambda v \bar{\lambda}$ is a $\vartheta$-palindrome beginning with $u a$ and strictly shorter than $(u a)^{\oplus}$, which is a contradiction.

We can naturally define a map $\psi_{\vartheta}: A^{*} \rightarrow A^{*}$ by $\psi_{\vartheta}(\varepsilon)=\varepsilon$ and

$$
\psi_{\vartheta}(u a)=\left(\psi_{\vartheta}(u) a\right)^{\oplus}
$$

for $u \in A^{*}, a \in A$. For any $u, v \in A^{*}$ one has $\psi_{\vartheta}(u v) \in \psi_{\vartheta}(u) A^{*} \cap A^{*} \psi_{\vartheta}(u)$, so that, as done for the iterated palindrome closure, the domain of $\psi_{\vartheta}$ can be extended to infinite words too. More precisely, if $x \in A^{\omega}$, then

$$
\psi_{\vartheta}(x)=\lim _{n \rightarrow \infty} \psi_{\vartheta}\left(w_{n}\right)
$$

where $\left\{w_{n}\right\}=\operatorname{Pref}(x) \cap A^{n}$ for all $n \geq 0$. The word $x$ is called the directive word of $\psi_{\vartheta}(x)$ and is denoted by $\Delta\left(\psi_{\vartheta}(x)\right)$. The images of infinite words over $A$ by $\psi_{\vartheta}$ have been called $\vartheta$-standard words in [6]. If $\vartheta=R$, then $\psi_{R}=\psi$, where $\psi$ is the iterated palindrome closure operator introduced in Section 1, so that an $R$-standard word is a standard episturmian word. A $\vartheta$-standard word, without specifying the antimorphism $\vartheta$, has been called pseudostandard word.

Example 3.2. Let $A=\{a, b\}$ and $\vartheta=E \circ R$, so that $\bar{a}=b$. For $x=(a b)^{\omega}$, we have $\psi_{\vartheta}(a)=a b, \psi_{\vartheta}(a b)=a b b a a b$, and

$$
s=\psi_{\vartheta}(x)=a b b a a b a b b a a b b a a b \ldots
$$

The word $s$ is the $\vartheta$-standard word having $x$ as its directive word.

The following theorem, proven in [6], shows that any $\vartheta$-standard word is a morphic image of the standard episturmian word having the same directive word.

Theorem 3.3. For any $w \in A^{\infty}$, one has $\psi_{\vartheta}(w)=\mu_{\vartheta}(\psi(w))$, where $\mu_{\vartheta}$ is the injective morphism defined as $\mu_{\vartheta}(a)=a^{\oplus}$ for any letter $a \in A$. 
For instance, one easily verifies that the word $s$ of Example 3.2 is equal to $\mu(f)$, where $f$ is the Fibonacci word and $\mu=\mu_{\vartheta}$ is the Thue-Morse morphism defined as $\mu(a)=a b, \mu(b)=b a$.

A new proof of Theorem 3.3 will be given in Section 4, as a consequence of a more general result. Some general properties of $\vartheta$-standard words have been considered in [6]. In particular, we recall that

Proposition 3.4. Let $s=\psi_{\vartheta}(x)$ be a $\vartheta$-standard word. The following hold:

(1) $w$ is a prefix of $s$ if and only if $w^{\oplus}$ is a prefix of $s$,

(2) the set of all $\vartheta$-palindromic prefixes of $s$ is given by $\psi_{\vartheta}(\operatorname{Pref}(x))$,

(3) $s$ is closed under $\vartheta$, i.e., if $w \in \operatorname{Fact}(s)$, then $\bar{w} \in \operatorname{Fact}(s)$.

Moreover, the following holds:

Proposition 3.5. If $s$ is a $\vartheta$-standard word over $A$ and two letters of $A$ occur infinitely often in $\Delta(s)$, then any prefix of $s$ is a left special factor of $s$.

Proof. A prefix $p$ of $s$ is also a prefix of any $\vartheta$-palindromic prefix $B$ of $s$ such that $|p| \leq|B|$. Since $B$ is a suffix of any $\vartheta$-palindromic prefix of $s$ whose length is at least $|B|$, and there exist two distinct letters (say $a$ and $b$ ) which occur infinitely often in $\Delta(s)$, by Proposition 3.4 one derives $B a, B b \in \operatorname{Fact}(s)$. Therefore, as $\bar{p} \in \operatorname{Suff}(B)$, we have $\bar{p} a, \bar{p} b \in \operatorname{Fact}(s)$, i.e., $\bar{p}$ is right special. Since by Proposition $3.4 s$ is closed under $\vartheta$, one has $\bar{a} p, \bar{b} p \in \operatorname{Fact}(s)$; as $\bar{a} \neq \bar{b}, p$ is left special.

For the converse of the previous proposition, we observe that a $\vartheta$-standard word $s$ can have left special factors which are not prefixes of $s$. For instance, consider the $\vartheta$-standard word $s$ in Example 3.2. As one easily verifies, $b$ and $b a$ are two left special factors of $s$, which are not prefixes.

However, we will show that if a left special factor $w$ of a $\vartheta$-standard word $s$ is not a prefix of $s$, then $|w| \leq 2$. For a proof of this we need a couple of lemmas. We denote by $A^{\prime}=A \backslash P A L_{\vartheta}$ the set of letters of $A$ that are not $\vartheta$-palindromic.

Lemma 3.6. The following holds:

$$
A^{\prime} \mu_{\vartheta}\left(A^{*}\right) \cap \mu_{\vartheta}\left(A^{*}\right)=\mu_{\vartheta}\left(A^{*}\right) A^{\prime} \cap \mu_{\vartheta}\left(A^{*}\right)=\emptyset .
$$

Proof. It is sufficient to observe that any word in $\mu_{\vartheta}\left(A^{*}\right)$ has an even number of occurrences of letters in $A^{\prime}$.

Lemma 3.7. Let $b, c \in A^{\prime}$, and let $f=\bar{b} \mu_{\vartheta}(u)$ and $g=\mu_{\vartheta}(v) c$ be factors of a $\vartheta$-standard word $t=\mu_{\vartheta}(s)$, with $s \in S E p$. Then:

(1) If bu, vc $\in \operatorname{Fact}(s)$ and $|f|>1$, then $f \neq g$.

(2) If $u \in \operatorname{Fact}(s)$ and $|f|>3$, then bu $\in \operatorname{Fact}(s)$.

Proof. (1) Since $|f|>1$, one has $u \neq \varepsilon$. By contradiction, if $f=g$, one has also $v \neq \varepsilon$, so that, from the definition of $\mu_{\vartheta}, \bar{b} b$ is a prefix of $\mu_{\vartheta}(v)$. Then $b \bar{b}$ is a prefix of $\mu_{\vartheta}(u)$, and so on; therefore, $f=\bar{b}(b \bar{b})^{k}=(\bar{b} b)^{k} \bar{b}$ for $k=|u|=|v| \geq 1$. Hence $c=\bar{b}, u=b^{k}$, and $v=\bar{b}^{k}$. As $k \geq 1$, by Proposition 1.6, bu $=b^{k+1}$ and $v c=\bar{b}^{k+1}$ cannot be both factors of the episturmian word $s$, a contradiction. 
(2) Since $|f|>3$, one derives $|u|>1$. By contradiction, suppose $b u \notin$ Fact $(s)$. By the preceding lemma and by Theorem 3.3, one derives $f=\mu_{\vartheta}\left(v^{\prime}\right) c^{\prime}$ for some suitable $v^{\prime} \in A^{*}$ and $c^{\prime} \in A^{\prime}$ such that $v^{\prime} c^{\prime} \in \operatorname{Fact}(s)$. As done before, one then obtains $f=(\bar{b} b)^{k} \bar{b}$ so that $b^{k}, \bar{b}^{k} \in \operatorname{Fact}(s)$, which is absurd by Proposition 1.6, as $k \geq 2$.

Theorem 3.8. Let $w$ be a left special factor of a $\vartheta$-standard word $t=\mu_{\vartheta}(s)$, with $s \in S E p$. If $|w| \geq 3$, then $w$ is a prefix of $t$.

Proof. By Theorem 3.3, $w$ can be written in one of the following ways:

(1) $w=\mu_{\vartheta}(u)$, with $u \in \operatorname{Fact}(s)$,

(2) $w=\bar{b} \mu_{\vartheta}(u)$, with $b u \in \operatorname{Fact}(s)$ and $b \in A^{\prime}$,

(3) $w=\mu_{\vartheta}(u) c$, with $u c \in \operatorname{Fact}(s)$ and $c \in A^{\prime}$,

(4) $w=\bar{b} \mu_{\vartheta}(u) c$, with buc $\in \operatorname{Fact}(s)$ and $b, c \in A^{\prime}$.

In case 1 , let $x w, y w \in \operatorname{Fact}(t)$ with $x \neq y$ letters of $A$. If $x$ is $\vartheta$-palindromic, then clearly one must have $x u \in \operatorname{Fact}(s)$. If $x \in A^{\prime}$, then by the preceding lemma one has $\bar{x} u \in \operatorname{Fact}(s)$, as $|x w|>3$. Since the same holds for $y, u$ is a left special factor of the episturmian word $s$, and therefore a prefix of it. Thus $w=\mu_{\vartheta}(u)$ is a prefix of $t$.

Cases 2 and 4 are absurd; indeed, by the preceding lemma one derives that every occurrence of $w$ is preceded by $b$.

Finally, in case 3 , by the preceding lemma one derives that every occurrence of $w$ is followed by $\bar{c}$. Hence $\mu_{\vartheta}(u c)$ is a left special factor of $t$ and one can apply the same argument as in case 1 to show that it is a prefix of $t$.

An infinite word $t$ is a $\vartheta$-word if there exists a $\vartheta$-standard word $s$ such that $\operatorname{Fact}(t)=\operatorname{Fact}(s)$. An $R$-word is an episturmian word.

Proposition 2.1 and Theorem 2.5 can be extended to the class of $\vartheta$-words, showing that if $w$ is a factor of a $\vartheta$-word, then $w^{\oplus}$ and $w^{\ominus}$ are also factors of $\vartheta$-words. A proof can be obtained as a consequence of Theorems 2.5 and 3.3 and of Corollary 2.6. However, we need the following lemma (cf. [6]):

Lemma 3.9. Let $u \in A^{*}$ and $x \in A \cup\{\varepsilon\}$. Then

$$
\left(\mu_{\vartheta}(u) x\right)^{\oplus}=\mu_{\vartheta}\left((u x)^{(+)}\right) .
$$

Theorem 3.10. Let $w$ be a factor of a $\vartheta$-standard word. Then each of $w^{\oplus}$ and $w^{\ominus}$ is a factor of a $\vartheta$-standard word.

Proof. We shall suppose $w \notin P A L_{\vartheta}$, otherwise the result is trivial. Since $w^{\ominus}=\bar{w}^{\oplus}$, it suffices to prove the result for $w^{\oplus}$. Let $A^{\prime}=A \backslash P A L_{\vartheta}$ as above. From Theorem 3.3, one derives that $w$ can be written in one of the following ways:

(1) $w=\mu_{\vartheta}(u) x$, with $x \in A \cup\{\varepsilon\}$ and $u x \in \operatorname{Fact}(E p)$,

(2) $w=\bar{a} \mu_{\vartheta}(u) b$, with $a, b \in A^{\prime}$ and $a u b \in \operatorname{Fact}(E p)$,

(3) $w=\bar{a} \mu_{\vartheta}(u)$, with $a \in A^{\prime}$ and $a u \in \operatorname{Fact}(E p)$. 
In the first case, by Theorem 2.5 there exists a standard episturmian word $s=\psi(\Delta)$ such that $(u x)^{(+)} \in \operatorname{Fact}(s)$. Thus, by Lemma 3.9 and Theorem 3.3, $w^{\oplus}=\mu_{\vartheta}\left((u x)^{(+)}\right)$is a factor of the $\vartheta$-standard word $\psi_{\vartheta}(\Delta)=\mu_{\vartheta}(s)$.

In the second case, by using Lemma 3.9, one has:

$$
w^{\oplus}=\bar{a}\left(\mu_{\vartheta}(u) b\right)^{\oplus} a=\bar{a} \mu_{\vartheta}\left((u b)^{(+)}\right) a \in \operatorname{Fact}\left(\mu_{\vartheta}\left(a(u b)^{(+)} a\right)\right) .
$$

Moreover, $a u b$ is not a palindrome, since otherwise one would derive, for instance using Lemma 3.9, that $w=\bar{a} \mu_{\vartheta}(u) b$ is a $\vartheta$-palindrome, which contradicts our assumption. Thus $(a u b)^{(+)}=a(u b)^{(+)} a$ and the result is a consequence of Theorem 3.3.

In the third case, since $w$ is not a $\vartheta$-palindrome, by Lemma 3.9 one obtains

$$
w^{\oplus}=\bar{a} \mu_{\vartheta}(u)^{\oplus} a \in \operatorname{Fact}\left(\mu_{\vartheta}\left(a u^{(+)} a\right)\right) .
$$

If $u=a^{k}$ for some $k \geq 0$, then $a u^{(+)} a=a^{k+2} \in \operatorname{Fact}(E p)$; otherwise $a u^{(+)}$is not a palindrome and $a u^{(+)} a=\left(a u^{(+)}\right)^{(+)}$, so that $a u^{(+)} a$ is episturmian by Corollary 2.6 and Theorem 2.5. Once again, the assertion follows from Theorem 3.3.

Corollary 3.11. Let $w$ be a factor of a $\vartheta$-standard word. Then there exists a $\vartheta$-standard word having both $w^{\oplus}$ and $w^{\ominus}$ as factors.

Proof. Trivial if $w \in P A L_{\vartheta}$. Let then $w=P b t=s a Q$, where $P$ (resp. $Q$ ) is the longest $\vartheta$-palindromic prefix (resp. suffix) of $w$, and $a, b \in A$. Thus $w \bar{a}$ and $\bar{b} w$, being respectively factors of $w^{\oplus}=s a Q \bar{a} \bar{s}$ and $w^{\ominus}=\bar{t} \bar{b} P b t$, are factors of $\vartheta$-standard words by Theorem 3.10 .

Suppose $w \bar{a} \notin P A L_{\vartheta}$. Then $(w \bar{a})^{\ominus}=a w^{\ominus} \bar{a}$, so that $w^{\ominus} \bar{a}$ is a factor of some $\vartheta$-standard word, by Theorem 3.10. Consider the word

$$
\left(w^{\ominus} \bar{a}\right)^{\oplus}=(\bar{t} \bar{b} P b t \bar{a})^{\oplus}=(\bar{t} \bar{b} s a Q \bar{a})^{\oplus},
$$

and call $Q^{\prime}$ the longest $\vartheta$-palindromic suffix of $w^{\ominus} \bar{a}$; then $Q^{\prime}=a Q \bar{a}$. Indeed, since $a Q \bar{a}$ is a $\vartheta$-palindrome, one has $\left|Q^{\prime}\right| \geq|a Q \bar{a}|$; but $|a Q \bar{a}|<\left|Q^{\prime}\right| \leq|s a Q \bar{a}|$ is absurd, for $Q$ would not be the longest $\vartheta$-palindromic suffix of $w$, and $\left|Q^{\prime}\right|>|s a Q \bar{a}|$ cannot happen, for otherwise there would exist a $\vartheta$-palindromic proper suffix of $w^{\ominus}$ having $w$ as a suffix, contradicting the definition of $w^{\ominus}$. Thus

$$
\left(w^{\ominus} \bar{a}\right)^{\oplus}=\bar{t} \bar{b} s a Q \bar{a} \bar{s} b t=\bar{t} \bar{b} P b t \bar{a} \bar{s} b t
$$

is a factor of some $\vartheta$-standard word, again by Theorem 3.10, and it contains both $w^{\oplus}$ and $w^{\ominus}$ as factors.

If $w \bar{a} \in P A L_{\vartheta}$ but $\bar{b} w \notin P A L_{\vartheta}$, one can prove by a symmetric argument that $\left(\bar{b} w^{\oplus}\right)^{\ominus}$ is a factor of some $\vartheta$-standard word having both $w^{\oplus}$ and $w^{\ominus}$ as factors. Let then $w \bar{a}, \bar{b} w \in P A L_{\vartheta}$, so that

$$
w^{\oplus}=w \bar{a}=a \bar{w} \text { and } w^{\ominus}=\bar{b} w=\bar{w} b .
$$


If $w$ is a single letter, one derives $w=a=b$, so that $w^{\oplus}=a \bar{a}$ and $w^{\ominus}=\bar{a} a$. Therefore $w^{\oplus}$ and $w^{\ominus}$ are factors of any $\vartheta$-standard word whose directive word begins with $a^{2}$. Let us then suppose $|w|>1$. From (2) it follows $w=a R b$ for some $R \in A^{*}$ such that $a R=\bar{R} \bar{a}=P$ and $R b=\bar{b} \bar{R}=Q$. Moreover,

$$
w=a R b=a \bar{b} \bar{R}=\bar{R} \bar{a} b,
$$

showing that $\bar{R}$ is a border of $w$. Therefore one has either $w=(a \bar{b})^{k}$ or $w=(a \bar{b})^{k} a$, for some $k>0$. In the first case, from (3) one derives $a=\bar{a}$ and $b=\bar{b}$, so that any $\vartheta$-standard word whose directive word begins with $a b^{k+1}$ contains both $w^{\oplus}=(a b)^{k} a$ and $w^{\ominus}=b(a b)^{k}$ as factors. In the latter case, by (3) one obtains $a=b$, so that any $\vartheta$-standard word whose directive word begins with $a^{k+1}$ contains both $w^{\oplus}=(a \bar{a})^{k}$ and $w^{\ominus}=(\bar{a} a)^{k}$ as factors.

Remark 3.12. For a finite episturmian word $w$, the proof of the preceding result can be simplified by using Theorem 2.5 and Corollary 2.6. Indeed, if $w$ is not a palindrome, we can write $w=P b t=s a Q$, where $P$ and $Q$ are respectively the longest palindromic prefix and suffix of $w$, and $a, b \in A$. By Theorem 2.5, $w^{(+)}$ and $w^{(-)}$are finite episturmian words; moreover $b w$ is a factor of $w^{(-)}$, so that by Corollary 2.6, $b w^{(+)}$is a finite episturmian word. By Theorem 2.5, $\left(b w^{(+)}\right)^{(-)}$ is a finite episturmian word, which has also $w^{(-)}$as a factor, as one can prove similarly as in the proof of Corollary 3.11.

In the case of Sturmian words, results analogous to Theorem 3.10 and Corollary 3.11 were proven in [6] with a different and simpler technique based on the structure of finite Sturmian words.

Example 3.13. Let $\tau$ be the Tribonacci word

$$
\tau=\psi\left((a b c)^{\omega}\right)=\text { abacabaabacababacabaabacabac } \ldots
$$

If $w=b a c \in \operatorname{Fact}(\tau)$, one has that $w^{(+)}=b a c a b$ and $w^{(-)}=c a b a c$ are factors of $\tau$. However, in the case of the factor $v=a b a c a b a b$, one has $v^{(+)}=a b a c a b a b a c a b a \in$ Fact $(\tau)$, whereas $v^{(-)}=$babacabab is not a factor of $\tau$, since otherwise $v$ would be a left special factor of $\tau$, which is a contradiction as $v \notin \operatorname{Pref}(\tau)$. Nevertheless, both $v^{(+)}$and $v^{(-)}$are factors of any episturmian word whose directive word begins with $a b c b b$. Indeed, $v=P b$ where $P=a b a c a b a$ is the longest palindromic prefix of $v$, and

$$
\left(b v^{(+)}\right)^{(-)}=\text {abacababacababacaba }=\psi(a b c b b) .
$$

\section{Words GENERATED BY NONEMPTY SEEDS}

We now consider a generalization of the construction of $\vartheta$-standard words. Define the map $\hat{\psi}_{\vartheta}: A^{*} \rightarrow A^{*}$ by setting $\hat{\psi}_{\vartheta}(\varepsilon)=u_{0}$ with $u_{0}$ a fixed word of $A^{*}$ called seed, and

for $u \in A^{*}$ and $a \in A$.

$$
\hat{\psi}_{\vartheta}(u a)=\left(\hat{\psi}_{\vartheta}(u) a\right)^{\oplus}
$$


As usual, we can extend this definition to infinite words $t \in A^{\omega}$ by:

$$
\hat{\psi}_{\vartheta}(t)=\lim _{n \rightarrow \infty} \hat{\psi}_{\vartheta}\left(w_{n}\right)
$$

where $\left\{w_{n}\right\}=\operatorname{Pref}(t) \cap A^{n}$ for all $n \geq 0$. The word $t$ is called the directive word of $\hat{\psi}_{\vartheta}(t)$, and denoted by $\Delta\left(\hat{\psi}_{\vartheta}(t)\right)$. When the seed $u_{0}$ is empty, one has $\hat{\psi}_{\vartheta}=\psi_{\vartheta}$ so that one obtains $\vartheta$-standard words. If $u_{0} \neq \varepsilon$, then any word $\hat{\psi}_{\vartheta}(t)$ is called $\vartheta$-standard with seed.

Example 4.1. Let $A=\{a, b, c\}, \vartheta$ be the involutory antimorphism exchanging $a$ and $b$ and fixing $c, u_{0}=a c b b c$, and $w=a b c$. Then

$$
\begin{aligned}
\hat{\psi}_{\vartheta}(w) & =\left(\hat{\psi}_{\vartheta}(a b) c\right)^{\oplus}=\left(\left(\hat{\psi}_{\vartheta}(a) b\right)^{\oplus} c\right)^{\oplus}=\left(\left((a c b b c a)^{\oplus} b\right)^{\oplus} c\right)^{\oplus} \\
& =\left((a c b b c a a c b b)^{\oplus} c\right)^{\oplus}=a c b b c a a c b b c a a c b c a c b b c a a c b b c a a c b .
\end{aligned}
$$

Let $t=x t_{1} t_{2} \ldots$, with $x \in A$ and $t_{i} \in A$ for $i \geq 1$. We remark that the set of $\vartheta$-palindromic prefixes of the word $w=\hat{\psi}_{\vartheta}(t)$ is

$$
\left(P A L_{\vartheta} \cap \operatorname{Pref}\left(u_{0}\right)\right) \cup\left\{u_{n} \mid n \geq 1\right\},
$$

where $u_{1}=\left(u_{0} x\right)^{\oplus}$ and $u_{i+1}=\left(u_{i} t_{i}\right)^{\oplus}$ for $i \geq 1$.

Define the endomorphism $\phi_{x}$ of $A^{*}$ by setting

$$
\phi_{x}(a)=\hat{\psi}_{\vartheta}(x a) \hat{\psi}_{\vartheta}(x)^{-1}
$$

for any letter $a \in A$. From the definition, one has that $\phi_{x}$ depends on $\vartheta$ and $u_{0}$; moreover, $\phi_{x}(a)$ ends with $\bar{a}$ for all $a \in A$, so that any word of the set $X=\phi_{x}(A)$ is uniquely determined by its last letter. Thus $X$ is a suffix code, and $\phi_{x}$ is an injective morphism.

Example 4.2. Let $A, \vartheta$, and $u_{0}$ be defined as in Example 4.1, and let $x=a$. Then

$$
\begin{aligned}
\phi_{a}(a) & =\hat{\psi}_{\vartheta}(a a) \hat{\psi}_{\vartheta}(a)^{-1}=a c b b c a a c b, \\
\phi_{a}(b) & =\hat{\psi}_{\vartheta}(a b) \hat{\psi}_{\vartheta}(a)^{-1}=a c b b c a, \\
\phi_{a}(c) & =\hat{\psi}_{\vartheta}(a c) \hat{\psi}_{\vartheta}(a)^{-1}=a c b b c a a c b c .
\end{aligned}
$$

To simplify the notation, in the following we shall often omit in the proofs the subscript $x$ from $\phi_{x}$, when no confusion arises.

Theorem 4.3. Fix $x \in A$ and $u_{0} \in A^{*}$. Let $\hat{\psi}_{\vartheta}$ and $\phi_{x}$ be defined as above. Then for any $w \in A^{*}$, the following holds:

$$
\hat{\psi}_{\vartheta}(x w)=\phi_{x}(\psi(w)) \hat{\psi}_{\vartheta}(x) .
$$


Proof. In the following we shall often use the property that if $\gamma$ is an endomorphism of $A^{*}$ and $v$ is a suffix of $u \in A^{*}$, then $\gamma\left(u v^{-1}\right)=\gamma(u) \gamma(v)^{-1}$.

We will prove the theorem by induction on $|w|$. It is trivial that for $w=\varepsilon$ the claim is true since $\psi(\varepsilon)=\varepsilon=\phi(\varepsilon)$. Suppose that for all the words shorter than $w$, the statement holds. For $|w|>0$, we set $w=v y$ with $y \in A$.

First we consider the case $|v|_{y} \neq 0$. We can then write $v=v_{1} y v_{2}$ with $\left|v_{2}\right|_{y}=0$, so that

$$
\hat{\psi}_{\vartheta}(x v)=\hat{\psi}_{\vartheta}\left(x v_{1} y v_{2}\right)=\hat{\psi}_{\vartheta}\left(x v_{1}\right) y \lambda=\bar{\lambda} \bar{y} \hat{\psi}_{\vartheta}\left(x v_{1}\right)
$$

for a suitable $\lambda \in A^{*}$. Note that $\hat{\psi}_{\vartheta}\left(x v_{1}\right)$ is the largest $\vartheta$-palindromic prefix (resp. suffix) followed (resp. preceded) by $y$ (resp. $\bar{y})$ in $\hat{\psi}_{\vartheta}(x v)$. Therefore,

$$
\hat{\psi}_{\vartheta}(x v y)=\bar{\lambda} \bar{y} \hat{\psi}_{\vartheta}\left(x v_{1}\right) y \lambda=\hat{\psi}_{\vartheta}(x v) \hat{\psi}_{\vartheta}\left(x v_{1}\right)^{-1} \hat{\psi}_{\vartheta}(x v) \text {. }
$$

By a similar argument one has:

$$
\psi(v y)=\psi(v) \psi\left(v_{1}\right)^{-1} \psi(v)
$$

By induction we have:

$$
\hat{\psi}_{\vartheta}(x v)=\phi(\psi(v)) \hat{\psi}_{\vartheta}(x), \quad \hat{\psi}_{\vartheta}\left(x v_{1}\right)=\phi\left(\psi\left(v_{1}\right)\right) \hat{\psi}_{\vartheta}(x) .
$$

Replacing in (5), and by (6), we obtain

$$
\begin{aligned}
\hat{\psi}_{\vartheta}(x v y) & =\phi(\psi(v)) \phi\left(\psi\left(v_{1}\right)\right)^{-1} \phi(\psi(v)) \hat{\psi}_{\vartheta}(x) \\
& =\phi\left(\psi(v) \psi\left(v_{1}\right)^{-1} \psi(v)\right) \hat{\psi}_{\vartheta}(x) \\
& =\phi(\psi(v y)) \hat{\psi}_{\vartheta}(x),
\end{aligned}
$$

which was our aim.

Now suppose that $|v|_{y}=0$ and $P A L_{\vartheta} \cap \operatorname{Pref}\left(u_{0} x\right) y^{-1} \neq \emptyset$. Let $\alpha_{y}$ be the longest word in $P A L_{\vartheta} \cap \operatorname{Pref}\left(u_{0} x\right) y^{-1}$, that is the longest $\vartheta$-palindromic prefix of $u_{0} x$ which is followed by $y$. Since $|v|_{y}=0$, one derives that the longest $\vartheta$-palindromic suffix of $\hat{\psi}_{\vartheta}(x v) y$ is $\bar{y} \alpha_{y} y$, whence

$$
\hat{\psi}_{\vartheta}(x v y)=\left(\hat{\psi}_{\vartheta}(x v) y\right)^{\oplus}=\hat{\psi}_{\vartheta}(x v) \alpha_{y}^{-1} \hat{\psi}_{\vartheta}(x v)
$$

By induction, this implies

$$
\hat{\psi}_{\vartheta}(x v y)=\phi(\psi(v)) \hat{\psi}_{\vartheta}(x) \alpha_{y}^{-1} \phi(\psi(v)) \hat{\psi}_{\vartheta}(x) .
$$

By using $(7)$ for $v=\varepsilon$, one has $\hat{\psi}_{\vartheta}(x y)=\hat{\psi}_{\vartheta}(x) \alpha_{y}^{-1} \hat{\psi}_{\vartheta}(x)$, and

$$
\phi(y)=\hat{\psi}_{\vartheta}(x y)\left(\hat{\psi}_{\vartheta}(x)\right)^{-1}=\hat{\psi}_{\vartheta}(x) \alpha_{y}^{-1}
$$


Moreover, since $\psi(v)$ has no palindromic prefix (resp. suffix) followed (resp. preceded) by $y$ one has

Thus from (8) we obtain

$$
\psi(v y)=\psi(v) y \psi(v) .
$$

$$
\begin{aligned}
\hat{\psi}_{\vartheta}(x v y) & =\phi(\psi(v)) \phi(y) \phi(\psi(v)) \hat{\psi}_{\vartheta}(x) \\
& =\phi(\psi(v) y \psi(v)) \hat{\psi}_{\vartheta}(x) \\
& =\phi(\psi(v y)) \hat{\psi}_{\vartheta}(x) .
\end{aligned}
$$

Finally we consider $|v|_{y}=0$ and $P A L_{\vartheta} \cap \operatorname{Pref}\left(u_{0} x\right) y^{-1}=\emptyset$. In this case, since $\hat{\psi}_{\vartheta}(x v)$ has no $\vartheta$-palindromic suffix preceded by $\bar{y}$ (has no $\vartheta$-palindromic prefix followed by $y$ ), we have

$$
\hat{\psi}_{\vartheta}(x v y)=\hat{\psi}_{\vartheta}(x v) y^{\oplus} \hat{\psi}_{\vartheta}(x v) .
$$

By induction we then obtain

$$
\begin{aligned}
\hat{\psi}_{\vartheta}(x v y) & =\hat{\psi}_{\vartheta}(x v) y^{\oplus} \hat{\psi}_{\vartheta}(x v) \\
& =\phi(\psi(v)) \hat{\psi}_{\vartheta}(x) y^{\oplus} \phi(\psi(v)) \hat{\psi}_{\vartheta}(x)
\end{aligned}
$$

In particular, if $v=\varepsilon$,

$$
\hat{\psi}_{\vartheta}(x y)=\hat{\psi}_{\vartheta}(x) y^{\oplus} \hat{\psi}_{\vartheta}(x),
$$

so

$$
\hat{\psi}_{\vartheta}(x y) \hat{\psi}_{\vartheta}(x)^{-1}=\hat{\psi}_{\vartheta}(x) y^{\oplus}=\phi(y) .
$$

Then from (11) and (9) one derives

$$
\begin{aligned}
\hat{\psi}_{\vartheta}(x v y) & =\phi(\psi(v)) \phi(y) \phi(\psi(v)) \hat{\psi}_{\vartheta}(x) \\
& =\phi(\psi(v) y \psi(v)) \hat{\psi}_{\vartheta}(x) \\
& =\phi(\psi(v y)) \hat{\psi}_{\vartheta}(x)
\end{aligned}
$$

which completes the proof.

Example 4.4. Let us refer to Example 4.1. We have $w=a b c, u_{0}=a c b b c$, and $\vartheta$ defined by $\bar{a}=b, \bar{c}=c$. By the preceding theorem, one has

$$
\hat{\psi}_{\vartheta}(a b c)=\phi_{a}(\psi(b c)) \hat{\psi}_{\vartheta}(a) .
$$

Since $\psi(b c)=b c b, \phi_{a}(b c b)=\phi_{a}(b) \phi_{a}(c) \phi_{a}(b)$, and $\hat{\psi}_{\vartheta}(a)=\left(u_{0} a\right)^{\oplus}=a c b b c a a c b$, by using (4) we obtain

$$
\hat{\psi}_{\vartheta}(a b c)=a c b b c a a c b b c a a c b c a c b b c a a c b b c a a c b,
$$

as already shown in Example 4.1. 
From Theorem 4.3, in the case that $w$ is an infinite word, we obtain:

Theorem 4.5. Let $w \in A^{\omega}$ and $x \in A$. Then

$$
\hat{\psi}_{\vartheta}(x w)=\phi_{x}(\psi(w))
$$

i.e., any $\vartheta$-standard word $s$ with seed is the image, by an injective morphism, of the standard episturmian word whose directive word is obtained by deleting the first letter of the directive word of $s$.

Proof. Let $w \in A^{\omega}, t=\psi(w)$, and $w_{n}=\operatorname{Pref}(w) \cap A^{n}$ for all $n \geq 0$. From Theorem 4.3 , for all $n \geq 0, \hat{\psi}_{\vartheta}\left(x w_{n}\right)=\phi\left(\psi\left(w_{n}\right)\right) \hat{\psi}_{\vartheta}(x)$. Since $\psi\left(w_{n+1}\right)=\psi\left(w_{n}\right) \xi_{n}$ with $\xi_{n} \in A^{+}$, one has $\phi\left(\psi\left(w_{n+1}\right)\right)=\phi\left(\psi\left(w_{n}\right)\right) \phi\left(\xi_{n}\right)$. Hence, $\hat{\psi}_{\vartheta}\left(x w_{n+1}\right)$ has the same prefix of $\hat{\psi}_{\vartheta}\left(x w_{n}\right)$ of length $\left|\phi\left(\psi\left(w_{n}\right)\right)\right|$, which diverges with $n$. Since

$$
\lim _{n \rightarrow \infty} \phi\left(\psi\left(w_{n}\right)\right)=\phi(\psi(w))
$$

the result follows.

In the case of an empty seed, from Theorem 4.3 one has

$$
\psi_{\vartheta}(x w)=\phi_{x}(\psi(w)) \psi_{\vartheta}(x)=\phi_{x}(\psi(w)) x^{\oplus} .
$$

Moreover, one easily derives that

$$
\phi_{x}(x)=x^{\oplus}, \quad \phi_{x}(y)=x^{\oplus} y^{\oplus} \text { for } y \neq x .
$$

When $u_{0}=\varepsilon$ and $\vartheta=R$, the morphism $\phi_{x}$ reduces to $\mu_{x}$ defined as $\mu_{x}(y)=x y$ for $y \neq x$ and $\mu_{x}(x)=x$. Since $x^{\oplus}=x$, from (12) one obtains the following formula due to Justin [9]:

$$
\psi(x w)=\mu_{x}(\psi(w)) x .
$$

It is noteworthy that Theorem 4.3 provides an alternate proof of Theorem 3.3:

Proof of Theorem 3.3. It is sufficient to observe that, in the case of an empty seed, $x^{\oplus}=\mu_{\vartheta}(x)$ and $\phi_{x}=\mu_{\vartheta} \circ \mu_{x}$, so that by (12) and (13) one derives:

$$
\psi_{\vartheta}(x w)=\left(\mu_{\vartheta} \circ \mu_{x}\right)(\psi(w)) \mu_{\vartheta}(x)=\mu_{\vartheta}\left(\mu_{x}(\psi(w)) x\right)=\mu_{\vartheta}(\psi(x w)),
$$

as desired.

Our next goal is to prove a result analogous to Theorem 3.8 for words generated by nonempty seeds. However, because of the presence of an arbitrary seed, one cannot hope to prove exactly the same assertion; thus in Theorem 4.9 we shall prove that any sufficiently long left special factor of a $\vartheta$-standard word with seed is a prefix of it, and give an upper bound for the minimal length from which this occurs, in terms of the length of $\left(u_{0} x\right)^{\oplus}$.

In the following, we shall set

$$
u_{1}=\hat{\psi}_{\vartheta}(x)=\left(u_{0} x\right)^{\oplus},
$$

so that $\phi_{x}(a)=\left(u_{1} a\right)^{\oplus} u_{1}^{-1}$ and $\left|\phi_{x}(a)\right| \leq\left|u_{1}\right|+2$ for any $a \in A$. 


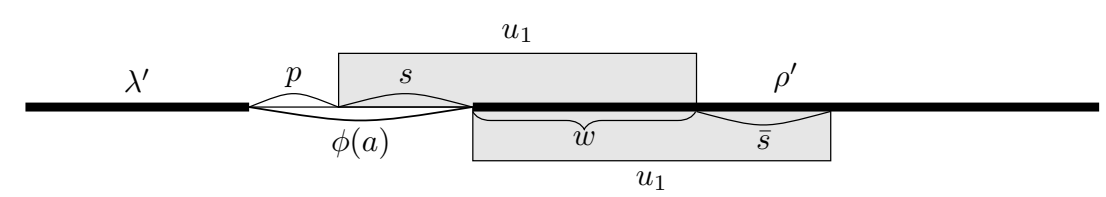

Figure 1. Proposition 4.7.

For any letter $a, u_{a}$ will denote (if it exists) the longest $\vartheta$-palindromic suffix (resp. prefix) of $u_{1}$ preceded (resp. followed) by $\bar{a}$ (resp. by $a$ ). One has then $u_{1}=\phi_{x}(a) u_{a}$ for any $a$ such that $u_{a}$ is defined, and $\phi_{x}(a)=u_{1} a^{\oplus}$ otherwise.

Lemma 4.6. Let $X=\phi_{x}(A)$. If $w \in X^{*}$, then $u_{1} \in \operatorname{Pref}\left(w u_{1}\right)$.

Proof. Trivial if $w=\varepsilon$. We shall prove by induction that for all $n \geq 1$, if $w \in X^{n}$, then $u_{1} \in \operatorname{Pref}\left(w u_{1}\right)$. Let $w \in X$. Then there exists $a \in A$ such that $w=\phi(a)=$ $\left(u_{1} a\right)^{\oplus} u_{1}^{-1}$. Thus $w u_{1}=\left(u_{1} a\right)^{\oplus}$, so that the statement holds for $n=1$.

Let us suppose the statement is true for $n$, we will prove it for $n+1$. If $w \in X^{n+1}$, there exist $a \in A$ and $v \in X^{n}$ such that $w=\phi(a) v$. By induction, $v u_{1}$ can be written as $u_{1} v^{\prime}$ for some $v^{\prime} \in A^{*}$. Then one has $w u_{1}=\phi(a) u_{1} v^{\prime}$ and, as shown above, $u_{1}$ is a prefix of $\phi(a) u_{1}$, which concludes the proof.

Recall ( $c f$. [2]) that a pair $(p, q) \in A^{*} \times A^{*}$ is synchronizing for the code $X$ over the alphabet $A$ if for all $\lambda, \rho \in A^{*}$,

$$
\lambda p q \rho \in X^{*} \Longrightarrow \lambda p, q \rho \in X^{*} .
$$

Proposition 4.7. The pair $\left(\varepsilon, u_{1}\right)$ is synchronizing for $X=\phi_{x}(A)$.

Proof. Since $X$ is a suffix code, it suffices to show that for any $\lambda, \rho \in A^{*}$,

$$
\lambda u_{1} \rho \in X^{*} \Longrightarrow u_{1} \rho \in X^{*} .
$$

This is trivial if $\lambda=\varepsilon$. Let us factorize $\lambda u_{1} \rho$ by the elements of $X$. Then we can write $\lambda=\lambda^{\prime} p$ and $u_{1} \rho=s \rho^{\prime}$, where $\lambda^{\prime}, \rho^{\prime} \in X^{*}$, and $p s=\phi(a) \in X$ for some letter $a$ (see Fig. 1). If $p=\varepsilon$, then trivially $u_{1} \rho \in X^{*}$. Suppose then $p \neq \varepsilon$, so that $s \notin X$.

Since $p s \in X$, it follows $|s| \leq\left|u_{1}\right|+1$. Let us prove that $|s| \leq\left|u_{1}\right|$. By contradiction, suppose $|s|=\left|u_{1}\right|+1$. Then $\phi(a)=p s=u_{1} a \bar{a}$ and $s=u_{1} \bar{a}$. Therefore $p s=u_{1} a \bar{a}=p u_{1} \bar{a}$, so that $u_{1} a=p u_{1}$. This implies $a=p$ and $u_{1}=a^{k}$ for a suitable $k>0$. Since $a$ is not a $\vartheta$-palindrome, it follows $u_{1} \notin P A L_{\vartheta}$, a contradiction.

Thus one has $u_{1}=s w$ for some $w \in \operatorname{Pref}\left(\rho^{\prime}\right)$. By Lemma 4.6, $u_{1}$ is a prefix of $\rho^{\prime} u_{1}$; clearly, $w$ is a prefix of $\rho^{\prime} u_{1}$ too. Therefore $w$ is a prefix of $u_{1}$, as $|w|=$ $\left|u_{1}\right|-|s|$. Thus $u_{1}=w \bar{s}$, and

$$
\left(u_{1} a\right)^{\oplus}=\phi(a) u_{1}=p s u_{1}=p s w \bar{s}=p u_{1} \bar{s} .
$$

Since $p \neq \varepsilon$, by Lemma 3.1 one obtains $\bar{s}=\varepsilon$. Hence $u_{1} \rho=\rho^{\prime} \in X^{*}$. 


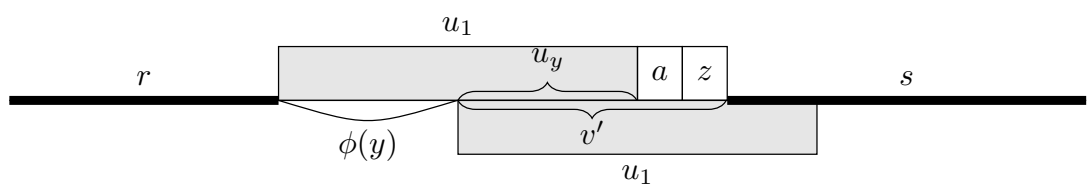

Figure 2. Lemma 4.8 .

In the following, if $Z$ is a finite subset of $A^{*}$, we shall denote by $Z^{\omega}$ the set of all infinite words which can be factorized by the elements of $Z$. As is well-known (cf. [2]) a word $t \in Z^{\omega}$ has a unique factorization by means of the elements of $Z$ if and only if $Z$ is a code having finite deciphering delay. By Lemma 4.6, the code $X=\phi_{x}(A)$ has the property that there exists an integer $n>0$ such that $u_{1} \in \operatorname{Pref}(v)$ for all $v \in X^{n}$; from Proposition 4.7 it follows that all pairs of $X^{n} \times X^{n}$ are synchronizing for $X$, so that $X$ has a bounded synchronization delay and therefore a finite deciphering delay.

Lemma 4.8. Let $X=\phi_{x}(A)$ and $w=r u_{1} a z s \in X^{*}$, with $a, z \in A$ and $r, s \in A^{*}$. If we set $v^{\prime}=\phi_{x}(a)^{-1} u_{1} a z$, then $\left(r, v^{\prime} s\right)$ is in $X^{*} \times X^{*}$ and it is an occurrence of $\phi_{x}(a)$ in $w$.

Proof. Let $w \in X^{*}$ be such that $w=r u_{1} a z s$, with $z \in A$. From Proposition 4.7 we have that $r$ and $u_{1} a z s$ are in $X^{*}$. Let $y \in A$ be a letter such that $v=\phi(y)^{-1} u_{1} a z s$ is in $X^{*}$ and set $v^{\prime}=\phi(y)^{-1} u_{1} a z$. It is clear from the definition of $\phi$ that either $v^{\prime}=\varepsilon, v^{\prime}=z$ or $v^{\prime}=u_{y} a z$, where $u_{y}$ is the longest $\vartheta$-palindromic suffix of $u_{1}$ preceded by $\bar{y}$. In the first two cases, it must be $\phi(y)=u_{1} a^{\oplus}$, so that $a=y$; let then $v^{\prime}=u_{y} a z$ (see Fig. 2). Since $v=v^{\prime} s \in X^{*}$, from Lemma 4.6 it follows that $u_{1}$ is a prefix of $v^{\prime} s u_{1}$, so $u_{y} a$, whose length is less than $\left|u_{1}\right|$, is a prefix of $u_{1}$. By definition, $u_{y}$ is a prefix of $u_{1}$ followed by $y$, hence $u_{y} y=u_{y} a$ and $a=y$. Thus $\left(r, v^{\prime} s\right) \in X^{*} \times X^{*}$ is an occurrence of $\phi(a)$ in $w$.

Theorem 4.9. Let $t=\hat{\psi}_{\vartheta}(x \Delta)$ be a $\vartheta$-standard word with seed. Then there exists an integer $N \geq 0$ such that any left special factor of $t$ of length greater than or equal to $N$ is a prefix of $t$.

Proof. Set $z=\psi(\Delta)=z_{1} z_{2} \ldots z_{n} \ldots$, where $z_{i} \in A$ for all $i \geq 1$. From Theorem 4.3 we have that $t=\phi(z)$, so that $t$ can be factorized uniquely as

$$
t=\phi\left(z_{1}\right) \phi\left(z_{2}\right) \ldots \phi\left(z_{n}\right) \ldots \in X^{\omega}
$$

where $X=\phi_{x}(A)$. We shall prove that each left special factor $w$ of $t$ longer than $2\left|u_{1}\right|+2$ is also a prefix of $t$. Since $w$ is left special, there exist two different occurrences of $w$ in $t$ preceded by distinct letters, say $a$ and $b$. Moreover, since $|w|>2\left|u_{1}\right|+2$, we can write

$$
w=p \phi\left(z_{i+1} \ldots z_{i+h}\right) s=p^{\prime} \phi\left(z_{j+1} \ldots z_{j+k}\right) s^{\prime},
$$


where $\phi\left(z_{i}\right)=\operatorname{rap}, \phi\left(z_{j}\right)=r^{\prime} b p^{\prime}, \phi\left(z_{i+h+1}\right)=s \lambda$, and $\phi\left(z_{j+k+1}\right)=s^{\prime} \lambda^{\prime}$, with $\lambda, \lambda^{\prime} \in A^{+}$and $i, j, h, k$ positive integers. Thus one can rewrite $t$ as

$$
t=\phi\left(z_{1} \ldots z_{i-1}\right) \operatorname{raw} \lambda \phi\left(z_{i+h+2} \ldots\right)=\phi\left(z_{1} \ldots z_{j-1}\right) r^{\prime} b w \lambda^{\prime} \phi\left(z_{j+k+2} \ldots\right) .
$$

Without loss of generality, we can suppose $|p| \leq\left|p^{\prime}\right|$. From (14) and from the preceding equation, we have

$$
\operatorname{rap}^{\prime} \phi\left(z_{j+1} \ldots z_{j+k}\right) s^{\prime} \lambda \phi\left(z_{i+h+2} \ldots\right) \in X^{\omega} .
$$

Since $|w|>2\left|u_{1}\right|+2$ and $p^{\prime} \leq\left|u_{1}\right|+1$, one has $\left|\phi\left(z_{j+1} \ldots z_{j+k}\right) s^{\prime}\right|>\left|u_{1}\right|+$ 1 , so that from Lemma 4.6, $u_{1}$ is a prefix of $\phi\left(z_{j+1} \ldots z_{j+k}\right) s^{\prime} \lambda^{\prime} u_{1}$ and then of $\phi\left(z_{j+1} \ldots z_{j+k}\right) s^{\prime}$.

By Proposition 4.7, $\left(p^{\prime}, \phi\left(z_{j+1} \ldots z_{j+k}\right) s^{\prime}\right)$ is a synchronizing pair for $X$, so that $r a p^{\prime}$ is in $X^{*}$. If $p^{\prime} \neq \varepsilon$, then $r^{\prime} b p^{\prime}$ is the only word of the code $X$ having $p^{\prime}$ as a suffix (recall that any codeword of $X$ is determined by its last letter); hence it should be a suffix of $r a p^{\prime}$, which is clearly a contradiction as $a \neq b$. Then $p^{\prime}=\varepsilon$, that implies also $p=\varepsilon$. Thus, we can write

$$
t=\phi\left(z_{1} \ldots z_{i}\right) w \lambda \phi\left(z_{i+h+2} \ldots\right)=\phi\left(z_{1} \ldots z_{j}\right) w \lambda^{\prime} \phi\left(z_{j+k+2} \ldots\right),
$$

and $z_{i} \neq z_{j}$, as $w$ is left special. Since

$$
w=\phi\left(z_{i+1} \ldots z_{i+h}\right) s=\phi\left(z_{j+1} \ldots z_{j+k}\right) s^{\prime}
$$

is longer than $2\left|u_{1}\right|+2$, and $|s|,\left|s^{\prime}\right| \leq\left|u_{1}\right|+1$, there exists a letter $c \in A$ such that $u_{1} c$ is a prefix of both $\phi\left(z_{i+1} \ldots z_{i+h}\right)$ and $\phi\left(z_{j+1} \ldots z_{j+k}\right)$. By Lemma 4.8 one has $\phi\left(z_{i+1} \ldots z_{i+h}\right)=\phi(c) \rho$ and $\phi\left(z_{j+1} \ldots z_{j+k}\right)=\phi(c) \rho^{\prime}$ with $\rho, \rho^{\prime} \in X^{*}$, so that $z_{i+1}=z_{j+1}=c$ since $X$ is a code.

Let $l$ be the greatest integer such that $z_{i+m}=z_{j+m}$ for all $m \leq l$. Then both $z_{i} z_{i+1} \ldots z_{i+l}$ and $z_{j} z_{j+1} \ldots z_{j+l}=z_{j} z_{i+1} \ldots z_{i+l}$ are factors of $z$. Since $z_{i} \neq z_{j}$, $z_{i+1} \ldots z_{i+l}$ is a left special factor of the episturmian word $z$, thus a prefix of $z$, i.e., $z_{i+1} \ldots z_{i+l}=z_{1} \ldots z_{l}$. Hence $\phi\left(z_{i+1} \ldots z_{i+l}\right)$ is a prefix of $t$.

Now let us suppose that $w^{\prime}=\phi\left(z_{i+l+1} \ldots z_{i+h}\right) s=\phi\left(z_{j+l+1} \ldots z_{j+k}\right) s^{\prime}$ is strictly longer than $u_{1}$. By Lemma 4.6, there exists a letter $d$ such that $u_{1} d$ is a prefix of $w^{\prime}$, so, by applying Lemma 4.8 to $w^{\prime} \lambda \in X^{*}$ and to $w^{\prime} \lambda^{\prime} \in X^{*}$ one derives $\phi\left(z_{i+l+1}\right)=\phi\left(z_{j+l+1}\right)=\phi(d)$, contradicting the fact that $i+l$ was the largest of such indexes. Then $\left|w^{\prime}\right| \leq\left|u_{1}\right|$. By Lemma 4.6, $u_{1}$ is a prefix of $w^{\prime} \lambda u_{1}$. Thus $w^{\prime}$ is a prefix of $u_{1}$ and $w=\phi\left(z_{i+1} \ldots z_{i+l}\right) w^{\prime}$ is a prefix of $\phi\left(z_{i+1} \ldots z_{i+l}\right) u_{1}=\phi\left(z_{1} \ldots z_{l}\right) u_{1}$. Let $m$ be an integer such that $\left|u_{1}\right| \leq$ $\left|\phi\left(z_{l+1} \ldots z_{l+m}\right)\right|$. By Lemma 4.6, $u_{1}$ is a prefix of $\phi\left(z_{l+1} \ldots z_{l+m}\right)$ and $\phi\left(z_{1} \ldots z_{l}\right) u_{1}$ is a prefix of $\phi\left(z_{1} \ldots z_{l+m}\right)$ which is a prefix of $t$. In conclusion, we obtain that $w$ is a prefix of $t$.

We observe that the proof of the preceding theorem shows that for a $\vartheta$-standard word $s$ with seed $u_{0}$, all left special factors of length greater than or equal to 
$N=2\left|u_{1}\right|+3$ are prefixes of $s$. However, this bound is not tight. In fact, for instance, if $u_{0}=\varepsilon$ then $N=5$, whereas from Theorem 3.8 one has that all left special factors of a $\vartheta$-standard word $s$, having length at least 3 , are prefixes of $s$.

In conclusion, we mention that some further general results on $\vartheta$-standard words with seed have been recently proved in [4].

Acknowledgements. The authors thank the anonymous referee for his/her valuable comments and suggestions.

\section{REFERENCES}

[1] V. Anne, L.Q. Zamboni and I. Zorca, Palindromes and pseudo-palindromes in episturmian and pseudo-palindromic infinite words, in Words 2005, number 36 in Publications du LaCIM, edited by S. Brlek and C. Reutenauer (2005) 91-100.

[2] J. Berstel and D. Perrin, Theory of Codes. Academic Press (1985).

[3] J. Berstel and P. Séébold, Sturmian words, in Algebraic Combinatorics on Words, edited by M. Lothaire. Cambridge University Press, Cambridge UK (2002). Chapter 2.

[4] M. Bucci, A. de Luca, A. De Luca and L.Q. Zamboni, On different generalizations of episturmian words. Theor. Comput. Sci., to appear.

[5] A. de Luca, Sturmian words: structure, combinatorics, and their arithmetics. Theor. Comput. Sci. 183 (1997) 45-82.

[6] A. de Luca and A. De Luca, Pseudopalindrome closure operators in free monoids. Theor. Comput. Sci. 362 (2006) 282-300.

[7] X. Droubay, J. Justin and G. Pirillo, Episturmian words and some constructions of de Luca and Rauzy. Theor. Comput. Sci. 255 (2001) 539-553.

[8] F. Durand, A characterization of substitutive sequences using return words. Discrete Mathematics 179 (1998) 89-101.

[9] J. Justin, Episturmian morphisms and a Galois theorem on continued fractions. RAIROTheor. Inf. Appl. 39 (2005) 207-215.

[10] J. Justin and G. Pirillo, Episturmian words and episturmian morphisms. Theor. Comput. Sci. 276 (2002) 281-313.

[11] L. Kari and K. Mahalingam, Watson-Crick conjugate and commutative words. Preliminary proceedings of DNA Computing 13, Memphis, USA. M.Garzon, H.Yan, Eds. (2007) 75-87.

Received January 9, 2007. Accepted December 4, 2007. 